\title{
MODELO MULTIOBJETIVO DE DEA PARA AVALIAR A SUSTENTABILIDADE DE EMPRESAS DE ENERGIA - APLICAÇÃO DA ABORDAGEM TBL + DEA
}

\author{
Mozart Caetano Heymann ${ }^{1}$ \\ mozart.mch@gmail.com \\ Rodrigo Goyannes Gusmão Caiado ${ }^{1}$ \\ rodrigoggcaiado@gmail.com \\ Lidia Angulo Meza ${ }^{1}$ \\ lidia.angulo.meza@gmail.com \\ Valdecy Pereira ${ }^{1}$ \\ valdecy.pereira@yahoo.com.br \\ Osvaldo Luís Gonçalves Quelhas ${ }^{1}$ \\ osvaldoquelhas@id.uff.br
}

${ }^{1}$ Universidade Federal Fluminense - Programa de Pós-Graduação Mestrado e Doutorado

Rua Passo da Pátria, 156 -Sala 309 -Bl. D -São Domingos, Niterói - RJ - CEP 24210-240

\section{RESUMO}

Este artigo objetiva usar um modelo multiobjetivo DEA para proporcionar flexibilidade adicional na busca de metas eficientes viáveis rumo ao desenvolvimento sustentável no setor de energia, permitindo que o tomador de decisão possa direcionar esforços mais adequados considerando as dimensões ambiental, social e econômica da sustentabilidade (Triple Bottom Line -TBL). Neste sentido, essa pesquisa baseia-se na aplicação do modelo de Otimização do Índice de Múltiplos Objetivos com Dominância (MORO-D), utilizado por Angulo Meza et al. (2018), dentro do contexto TBL + DEA. Assim, é apresentado um estudo de caso com dados reais de sete empresas brasileiras de energia elétrica durante o período 2015-2017, 21 unidades tomadoras de decisão (DMUs), com foco simultâneo na redução das despesas de capital (OPEX), no aumento do número de consumidores e na redução da emissão de gases do efeito estufa.

Palavras chave: Modelos DEA multiobjetivo; TBL + DEA; Sustentabilidade.

\section{ABSTRACT}

This paper aims to use a multi-objective DEA model to provide additional flexibility in pursuing viable, efficient goals towards sustainable development in the energy sector, allowing the decision maker to address more appropriate efforts, taking into account the environmental, social and economic dimensions of sustainability (Triple Bottom Line TBL). In this sense, this research is based on the application of the Multiple Objective Ratio Optimization with Dominance (MORO-D), used by Angulo Meza et al. (2018), within the $\mathrm{TBL}+$ DEA context. Thus, a case study with real data from seven Brazilian electricity companies during the period 2015-2017, 21 decision-making units (DMUs), with simultaneous focus on reducing capital expenditures (OPEX), number of consumers and in reducing the emission of greenhouse gases.

Keywords: Multiobjective DEA models; TBL + DEA; Sustainability.

\section{Como Citar:}

HEYMANN, Mozart Caetano; CAIADO, Rodrigo Goyannes Gusmão; ANGULO MEZA, Lidia; PEREIRA, Valdecy; QUELHAS, Osvaldo Luis Gonçalves. Modelo Multiobjetivo de DEA para Avaliar a Sustentabilidade de Empresas de Energia - aplicação da abordagem TBL + DEA. In: SIMPÓSIO DE PESQUISA OPERACIONAL E LOGÍSTICA DA MARINHA, 19., 2019, Rio de Janeiro, RJ. Anais [...]. Rio de Janeiro: Centro de Análises de Sistemas Navais, 2019. 


\section{INTRODUÇÃO}

Com a alta competitividade e o aumento da consciência da população, as organizações passaram a dar mais atenção à sustentabilidade, melhorando seus processos de forma a reduzir os custos, desperdícios e o consumo de recursos naturais como insumo para a produção, buscando minimizar o impacto no meio ambiente e atender as necessidades e expectativas dos clientes [1]. Nesta direção em 2015, os Objetivos de Desenvolvimento Sustentável (ODS) definiram a Agenda 2030, para o desenvolvimento sustentável, e representam um grande esforço multilateral para mudar o mundo para caminhos mais sustentáveis e resilientes [2]. A sustentabilidade é um conceito multidimensional [3] composto pelas dimensões ambiental, social e econômica, também conhecido como Triple Bottom Line (TBL) [4]. O conceito do TBL busca o equilíbrio entre o incremento econômico, gestão ambiental e igualdade social, para alcançar o desenvolvimento sustentável, cujos resultados de uma organização são medidos segundo as três dimensões [5].

A importância crescente das três dimensões do TBL acarretou a implementação de metodologias que pudessem mensurá-las, tais como o Retorno sobre o Investimento Sustentável (Sustainable Return on Investment - SROI), que atribui valores monetários aos impactos ambientais e sociais [6]. Neste contexto é sustentado por [7], que a estrutura de comunicação voluntária do desempenho sustentável das empresas mais conhecida em todo o mundo é o Global Reporting Initiative (GRI), e através desses relatórios de sustentabilidade, diversas organizações apresentam os impactos TBL de suas atividades, medindo, compreendendo e comunicando seu desempenho. Tais relatórios representam, assim, uma importante fonte de informação, que pode ser utilizada para a avaliação comparativa do desempenho de empresas e organizações [5].

Como uma metodologia para realizar comparações de desempenho, ou seja, apuração da eficiência, em [8] é apresentada a análise envoltória de dados (Data Envelopment Analysis - DEA) que é uma ferramenta que vem sendo empregada para analisar diversas questões corporativas, regionais e nacionais, ligadas à sustentabilidade. $\mathrm{O}$ método avalia a performance de Unidades de Produção (Decision Making Units - DMUs) através de múltiplas medidas de desempenho organizadas como entradas (inputs) e saídas (outputs), utilizando modelos de programação matemática para fazer a referida avaliação, e assim fornecendo um índice de eficiência para cada DMU, sendo esse índice igual a 1 para as eficientes e menor que 1 para ineficientes [9]. Além do índice de eficiência, DEA fornece os alvos para as DMUs ineficientes, correspondendo a esses alvos os valores de inputs e outputs que devem ser atingidos para essas DMUs se tornarem eficientes, e fornece também os benchmarks como referência para melhores práticas. Diversos modelos têm sido propostos em DEA para lidar com as diferentes particularidades ou necessidades dos estudos de caso, dentre eles, os modelos multiobjetivos têm sido utilizados, por exemplo, para identificar um alvo preferido na fronteira eficiente com uma abordagem interativa [10], ou para aumentar a discriminação DEA quando muitas unidades são classificadas como totalmente eficientes [11]. Por outro lado, como mencionado em [12], os modelos DEA convencionais determinam apenas um alvo para cada DMU ineficiente, ou seja, níveis a serem atingidos para alcançarem a eficiência, podendo ocorrer, no entanto, a possibilidade de que esse alvo não seja viável do ponto de vista operacional ou gerencial. Assim, em [13] denota-se uma forma de fornecer um conjunto de alvos alternativos para uma DMU ineficiente utilizando um modelo multiobjetivo DEA chamado "Otimização do Índice de Múltiplos Objetivos com Dominância" (Multiple Objective Ratio Optimization with Dominance - MORO-D). Entre as vantagens do modelo MORO-D em relação ao DEA padrão, é permitir mais flexibilidade e possibilidade de ter um conjunto de alvos alternativos para cada unidade ineficiente [12]. 
Portanto, o objetivo deste artigo é usar o modelo MORO-D dentro do contexto TBL + DEA, para realizar a avaliação da sustentabilidade de empresas brasileiras de energia durante o período 2015-2017. Foram usados dados reais dos indicadores das três perspectivas do TBL contidos nos relatórios de sustentabilidade, seguindo o modelo GRI, deste grupo de empresas, a fim de demonstrar a flexibilidade que o modelo multiobjetivo fornece. Desta forma, a utilização de um modelo multiobjetivo DEA que proporcione alvos alternativos para melhorar a sustentabilidade corporativa no setor de energia, propicia flexibilidade adicional na busca de metas eficientes viáveis no processo de tomada de decisão, permitindo que o decisor possa escolher um alvo adequado, considerando suas necessidades / limitações operacionais e gerenciais, e, assim, definam o curso de ações mais condizentes de acordo com suas condições e recursos. Como o modelo MORO-D fornece as metas e não o índice de eficiência, nesse estudo foi adotado o índice de eficiência proposto em [14], que vem sendo utilizado em outros trabalhos [12].

Dessa forma, o presente artigo possui as seguintes contribuições para a literatura de DEA: (i) é o primeiro artigo a usar um modelo multiobjetivo DEA para determinar alvos alternativos para DMUs ineficientes no setor de energia; (ii) é uma extensão dos trabalhos anteriores de TBL + DEA, oferecendo as empresas de energia elétrica uma ferramenta de medição do desempenho sustentável baseada em programação linear multiobjetivo no contexto TBL da sustentabilidade corporativa, contribuindo assim, para uma melhor análise e apoio à decisão, podendo direcionar esforços rumo à redução de impactos socioambientais e a melhora no valor econômico nas empresas de energia.

Este artigo está organizado da seguinte maneira. A seção 2 define a metodologia da pesquisa e a modelagem utilizada. A seção 3 é dedicada a estabelecer o estudo de caso, a descrever a amostra utilizada e a justificativa de escolha das variáveis. A seção 4 apresenta e discute os resultados do modelo DEA utilizado para realizar a análise de desempenho sustentável das empresas de energia. Por fim, a seção 5 conclui e apresenta sugestões para pesquisas futuras.

\section{METODOLOGIA}

\subsection{Análise Envoltória de Dados - DEA}

DEA é um método fundamentado em programação linear para medição de eficiência com base no trabalho original de [15] que foi posteriormente popularizado por [9]. Uma das principais vantagens desta análise é não exigir nenhuma suposição prévia sobre as relações funcionais subjacentes entre insumos e produtos [16]. O modelo CRS (do inglês Constant Returns to Scale), é também nomeado com as iniciais de seus autores, modelo CCR (Charnes, Cooper e Rhodes). Em [9] foi proposta uma superfície linear não paramétrica e por partes - a fronteira de eficiência - para calcular a eficiência das DMUs. A principal característica deste modelo é o retorno constante à escala, ou seja, a variação das saídas é proporcional à variação dos insumos. DEA por estar fundamentado em programação linear, admite para um modelo primal ou dos multiplicadores, o respectivo dual ou do envelope. Como exemplo considerando $n$ DMUs, cada uma com " $p$ " inputs $(x)$ e " $k$ " outputs $(y) ; v_{p}$ e $u_{k}$ representam os pesos dados aos inputs $i$ e aos outputs $j$, respectivamente, a eficiência relativa de cada DMU pode ser calculada com o modelo.dos multiplicadores (1):

$$
\begin{aligned}
\operatorname{Max} & \frac{\sum u_{k} y_{k i}}{\sum v_{p} x_{p i}} \\
\text { Sujeito a: } & \frac{\sum u_{k} y_{k j}}{\sum v_{p} x_{p j}} \leq 1, \forall j \\
u_{k} \geq 0, & v_{p} \geq 0, \quad \forall k e \forall p
\end{aligned}
$$


Em 1984, conforme [17] é apresentado um novo modelo DEA clássico, diferente do modelo CCR, denominado como modelo VRS (do inglês Variable Return of Scale) que também é conhecido pelas iniciais dos seus autores como modelo BCC (Banker, Charnes and Cooper). O modelo $\mathrm{BCC}$ considera retornos variáveis, ou seja, não há proporcionalidade entre entradas e saídas, o que significa que um incremento na variável de input não necessariamente provoca um incremento proporcional na variável de output. Como exemplo desse modelo BCC, orientado a input, pode ser representado pelo modelo do envelope (2),

$$
\begin{aligned}
& \text { Min } h_{0} \\
& \quad \text { Sujeito } a \text { : } \\
& \sum_{k} \lambda_{j} x_{j 1} \leq h_{0} x_{i 0} \\
& \sum_{k} \lambda_{j} y_{j 1} \leq y_{j 0} \\
& \sum_{k} \lambda_{k}=1
\end{aligned}
$$

Em que

$$
\lambda_{k} \geq 0, \forall k
$$

$\lambda_{k} \geq 0, \forall k$, sendo $\lambda$ um vetor de variáveis e $h_{0} h_{0}$ representa um indicador de eficiência técnica onde $h_{0} h_{0} \in[0,1]$

Além disso, também foi observado que o modelo CCR identifica apenas a eficiência técnica, supondo que todas as DMUs estejam trabalhando em grande escala. Já o modelo BCC identifica a diferença entre eficiência técnica e eficiência de escala [17]. Outro ponto em DEA é a orientação do modelo. Se o modelo é orientado a input, a função objetivo leva a minimizar as entradas mantendo as saídas como constantes. No entanto, se o modelo for orientado a output, a função objetivo leva a maximizar as saídas mantendo as entradas como constantes. Em ambos os modelos, as DMUs devem ser homogêneas, ou seja, devem realizar tarefas semelhantes e devem ter o mesmo objetivo, bem como, relação causal entre as variáveis [19].

\subsection{DEA - Variável Indesejada}

Os modelos clássicos de DEA ao serem elaborados e posteriormente calculados, para obtenção das eficiências das DMUs, têm como premissa básica a maximização dos produtos, outputs, ou a minimização dos insumos, inputs. Entretanto, nem sempre a variável tem o comportamento conforme descrito anteriormente, pois podem ocorrer resultados do processo produtivo, que não sejam desejáveis, e, portanto, a sua produção deve ser minimizada, ao invés de ser maximizada.

Tais variáveis são denominadas outputs indesejáveis [20], como um exemplo notório tem-se a emissão de gases poluentes em processos industriais. Nesses casos para executar o cálculo da eficiência das unidades produtivas de forma correta é necessário realizar o tratamento adequado dessas variáveis, e basicamente existem quatro técnicas, objetivando a adequação dessas variáveis [21], de tal forma que possam ser utilizadas de forma assertiva em uma modelagem DEA. Neste estudo os outputs indesejáveis serão incorporados no modelo como inputs (INP). Denota-se como um caminho natural, pois com 
esse procedimento de tratar o output indesejável como input, quanto menor for a sua produção, melhor será o desempenho da DMU [20].

\subsection{Modelo DEA multiobjetivo MORO-D}

Por definição, todos os alvos estão localizados na fronteira eficiente, isto significa que, se as DMUs ineficientes alcançarem uma de suas metas alternativas, será eficiente. $\mathrm{O}$ modelo multiobjetivo pela sua natureza permite obter um conjunto de alvos, simultaneamente, considerando diferentes dimensões, ou seja, cada variável individual pode ser otimizada em diferentes proporções. Como mencionado anteriormente, esta é uma vantagem sobre os modelos padrão DEA, pois como o modelo MORO-D fornece um conjunto de metas para cada DMU ineficiente, permite que seja adotada, dentre as alternativas obtidas, a ação mais adequada de acordo com as condições e recursos disponíveis. O modelo MORO-D é apresentado em [22] e [13], considerando retornos variáveis à escala (VRS) e restrições de dominância (D), conforme modelo (3).

$$
\begin{aligned}
& \operatorname{Max} \phi_{1} \\
& \operatorname{Max} \phi_{s} \\
& \operatorname{Min} \varphi_{1} \\
& \operatorname{Min} \varphi_{m} \\
& \text { subject to } \\
& \sum_{\mathrm{j}=1}^{\mathrm{n}} \mathrm{y}_{\mathrm{rj}} \lambda_{\mathrm{j}} \geqslant \phi_{\mathrm{r}} \mathrm{y}_{\mathrm{ro}} ; \forall \mathrm{r}=1, \ldots, \mathrm{S} \\
& \sum_{\mathrm{j}=1}^{\mathrm{n}} \mathrm{x}_{\mathrm{ij}} \lambda_{\mathrm{j}} \leqslant \varphi_{i} x_{\mathrm{io}} ; \forall i=1, \ldots, \mathrm{m} \\
& \sum_{j=1}^{n} \lambda_{j}=1 \\
& \phi_{r} \geqslant 1 ; \forall \mathrm{r}=1, \ldots, \mathrm{S} \\
& \varphi_{i} \leqslant 1 ; \forall i=1, \ldots, \mathrm{m} \\
& \phi_{r}, \varphi_{i}, \lambda_{\mathrm{j}} \geqslant 0
\end{aligned}
$$

Neste modelo para $m$ entradas, $s$ saídas e $n$ DMUs, $x_{i j}$ é a entrada $\mathrm{i}$ de $D M U j$, $\mathrm{i}=1, \ldots, \mathrm{m} ; \mathrm{y}_{\mathrm{rj}}$ é a saída $\mathrm{r}$ de $\mathrm{DMUj}, \mathrm{r}=1, \ldots \mathrm{s}, \mathrm{com} \mathrm{j}=1, \ldots, \mathrm{n} ; \lambda_{\mathrm{j}}$ é a intensidade da contribuição do benchmark j para o alvo do DMU em avaliação $\left(\mathrm{DMU}_{\mathrm{o}}\right)$. Os múltiplos objetivos asseguram a otimização com o aumento de saídas $\left(\phi_{\mathrm{r}}\right)$ e a diminuição de insumos $\left(\varphi_{i}\right)$, enquanto as restrições de cada variável, garantem que esses novos níveis estejam localizados na fronteira eficiente. A soma de $\lambda_{\mathrm{j}}$ igual a um, caracteriza VRS como em qualquer modelo DEA. As duas últimas restrições garantem que os níveis de produção sejam mantidos ou aumentados e que os níveis de entrada sejam mantidos ou diminuídos; isso é chamado de domínio (D). Se, por qualquer particularidade do processo, as entradas puderem ser aumentadas ou saídas diminuídas, essas duas restrições deverão ser removidas.

Conforme descrito em [12], ao resolver este modelo, se a DMU observada for eficiente, então todos $\varphi_{\mathrm{i}}$ e $\varphi_{\mathrm{r}}$ serão iguais a 1; se não, a DMU é ineficiente e um conjunto de soluções ótimas para $\varphi_{i}^{*}$ e $\varphi_{r}^{*}$ será obtido.

Os alvos são calculados conforme as equações. (4) e (5). 
Diferentemente dos modelos padrão DEA, o modelo MORO-D não fornece um índice de eficiência associado a cada alvo. Portanto, o índice (h) proposto por [14], é usado para determinar o índice de eficiência para cada alvo, que está associado ao modelo MOROD. Este índice, apresentado através da equação (6), utiliza as variáveis individuais para cada entrada $\left(\varphi_{\mathrm{i}}\right)$ e cada saída $\left(\phi_{\mathrm{r}}\right)$ fornecida pelo modelo MORO- D.

$$
h=1-\sqrt{\frac{1}{m} \sum_{i=1}^{m}\left(1-\varphi_{i}\right)^{2}+\frac{1}{s} \sum_{r=1}^{s}\left(1-\frac{1}{\phi_{r}}\right)^{2}}
$$

Existem outros índices de eficiência que podem ser usados, que se baseiam em médias aritméticas ou ponderadas, conforme [14], mas não serão objeto de estudo neste trabalho.

\section{ESTUDO DE CASO}

O estudo de caso tem por objeto a análise do desempenho sustentável de empresas brasileiras de energia, no ciclo de 3 anos - 2015 a 2017, com uso do modelo DEA multiobjetivo (MORO-D). Assim sendo, foram selecionadas empresas do setor aderentes ao Global Reporting Initiative (GRI), cujos indicadores encontram-se reportados nos respectivos relatórios, e que concomitantemente estivessem listadas na carteira do Índice de Sustentabilidade Empresarial (ISE), divulgado anualmente pela BM\&F BOVESPA, como sendo empresas detentoras das melhores práticas em sustentabilidade, tendo esse índice o condão de atuar como indutor de boas práticas no meio empresarial brasileiro e ser uma referência para o investimento socialmente responsável [23]. Ressalta-se que no período em tela, a carteira ISE teve no mínimo oito empresas do setor que corresponde a $20 \%$ da carteira anual, demonstrando representatividade do setor no campo da sustentabilidade. Dessas oitos empresas do setor elétrico, sete delas estiveram presentes em todas as edições da carteira ISE do período sob estudo, sendo estas empresas selecionadas para a presente pesquisa. As características destas sete Empresas de Energia (Ej), encontram-se elencadas a seguir:

- Empresa 1 - (E1) - Atuante nas áreas de geração, distribuição e comercialização de energia, além de prestar serviços de soluções de energia. Na área de geração de energia conta com 6 usinas hidrelétricas e 1 usina eólica, com capacidade instalada de aproximadamente 1,0 GW em operação.

- Empresa 2 - (E2) - Atuante nas áreas de geração, distribuição e comercialização de energia, além de prestar serviços de soluções de energia. Na área de geração de energia conta com 12 usinas hidrelétricas, com capacidade instalada de 2,7 GW em operação.

- Empresa 3 - (E3) - Atuante nas áreas de geração, transmissão e distribuição de energia, além de serviços em telecomunicações. Na área de geração de energia conta com 20 usinas (sendo 18 hidrelétricas, 1 termelétrica e 1 eólica), com capacidade instalada de 4,8 GW em operação

- Empresa 4 - (E4) - Atuante nas áreas de geração, transmissão e distribuição de energia, além de distribuição de gás natural, serviços em telecomunicações e eficiência energética. $\mathrm{Na}$ área de geração de energia conta com 121 usinas (86 hidre- 
létricas, 3 termelétricas e 32 eólicas), capacidade instalada de 8,5 GW.

- Empresa 5 - (E5) - Atuante nas áreas de geração, transmissão e distribuição de energia, além de serviços em eficiência energética. Na área de geração de energia conta com 180 usinas (sendo 45 hidrelétricas, e 125 termelétricas, 8 eólicas e 2 nucleares), com capacidade instalada de $43 \mathrm{GW}$.

- Empresa 6 - (E6) - Atuante nas áreas de geração, transmissão e distribuição de energia, além de serviços em telecomunicações e em eficiência energética. Na área de geração de energia conta com 9 usinas (sendo 46 hidrelétricas, e 9 termelétricas, 33 eólicas e 1 solar), com capacidade instalada de $7 \mathrm{GW}$.

- Empresa 7 - (E7) - Atuante nas áreas de geração e distribuição de energia, além de serviços em soluções de energia elétrica no país. Na área de geração de energia conta com 16 usinas (sendo 7 hidrelétricas, 8 pequenas centrais hidrelétricas e 1 termelétrica), com capacidade instalada de 3,7 GW.

Estas sete empresas serão objeto de estudo, associado a cada ano do período determinado de três anos, correspondendo a 21 DMUs que serão analisadas pelo modelo DEA multiobjetivo MORO-D.

Foi utilizado um indicador para cada dimensão do TBL, que são as variáveis do modelo, buscando-se na literatura de DEA relacionado com sustentabilidade, as variáveis que tivessem ligações direta com as operações das empresas do setor e relevância para a sociedade em geral. A seguir a descrição das variáveis adotadas.

- Ambiental: Emissões diretas de gases de efeito estufa - GEE, correspondem às emissões de gases danosos ao meio ambiente e aos seres vivos, oriundas de fontes de propriedade ou controlada pela empresa [24]. É um produto resultante das operações das empresas, mas por ser nocivo à saúde trata-se de um output indesejável, cujo objetivo é reduzir a sua produção. É expresso em tCO2e (toneladas de carbono equivalente).

- Social: Número de consumidores corresponde ao número de consumidores atendidos pela empresa no período determinado, trata-se de um output de dimensão operacional, mas também se encontra vinculada ao aspecto social, pois a energia elétrica é um insumo primordial para a sociedade. Portanto, com a expansão da cartela de clientes das empresas de distribuição, espera-se que o acesso à energia aumente e, do ponto de vista social, as companhias auxiliem na distribuição desse bem primordial para o desenvolvimento das comunidades. Ademais, inúmeros artigos que visam avaliar a eficiência de distribuidoras de energia através de modelos DEA usam essa variável como output do modelo.

- Econômica: OPEX sigla derivada da expressão inglesa "Operational Expenditure”, que significa o capital utilizado para manter ou melhorar os bens físicos de uma empresa, são os custos e despesas operacionais. Portanto uma variável econômica, correspondendo a um input, que uma vez assegurado o mesmo nível de produção, tende a reduzir, na medida que, a empresa mantém seus bens funcionando observando a boa técnica, além de ser aplicada como dimensão econômica em avaliações de sustentabilidade de corporações, através de modelos DEA [8]. 
A coleta de dados foi realizada por meio dos relatórios anuais de sustentabilidade das empresas e da Agência Nacional de Energia Elétrica [25], disponíveis nos seus respectivos sites.

Na Tabela 1 encontram-se os 3 indicadores de sustentabilidade (variáveis) elencados para cada empresa-ano (DMUs).

Tabela 1 - DMUs com as respectivas Variáveis e Variações anuais das empresas para cada variável

\begin{tabular}{lcrrrrrr}
\hline \multicolumn{1}{c}{ DMUs } & \multicolumn{7}{c}{ VARIÁVEIS } \\
\hline EMPRESAS & ORDEM & \multicolumn{2}{c}{ ECONOMMICO } & AMBIENTAL & SOCLAL \\
\cline { 3 - 8 } ANOS & & OPEX) & $\begin{array}{c}\Delta \% \\
\text { anual }\end{array}$ & $\begin{array}{c}\text { EMISSÕES } \\
\text { (tCO2e) }\end{array}$ & $\begin{array}{c}\Delta \% \\
\text { anual }\end{array}$ & $\begin{array}{c}\text { CONSUMIDORES } \\
\text { (QUANTIDADE) }\end{array}$ & $\begin{array}{c}\Delta \% \\
\text { anual }\end{array}$ \\
\hline E1-2015 & 1 & 9.885 & - & 31.557 & - & 3.403 .146 & - \\
E2-2015 & 2 & 12.093 & - & 6.156 & - & 6.522 .904 & - \\
E3-2015 & 3 & 12.912 & - & 231.519 & - & 3.437 .021 & - \\
E4-2015 & 4 & 18.318 & - & 164.537 & - & 6.382 .796 & - \\
E5-2015 & 5 & 31.026 & - & 8.413 .812 & - & 3.353 .822 & - \\
E6-2015 & 6 & 17.954 & - & 798.633 & - & 7.801 .799 & - \\
E7-2015 & 7 & 8.975 & - & 4.529 .463 & - & 2.685 .324 & - \\
\hline E1-2016 & 8 & 8.268 & $-16 \%$ & 38.727 & $23 \%$ & 3.327 .065 & $-2 \%$ \\
E2-2016 & 9 & 10.123 & $-16 \%$ & 22.690 & $269 \%$ & 6.388 .821 & $-2 \%$ \\
E3-2016 & 10 & 11.279 & $-13 \%$ & 282.745 & $22 \%$ & 3.320 .114 & $-3 \%$ \\
E4-2016 & 11 & 15.903 & $-13 \%$ & 15.462 & $-91 \%$ & 6.195 .904 & $-3 \%$ \\
E5-2016 & 12 & 45.910 & $48 \%$ & 6.954 .216 & $-17 \%$ & 3.229 .964 & $-4 \%$ \\
E6-2016 & 13 & 16.589 & $-8 \%$ & 527.117 & $-34 \%$ & 7.544 .571 & $-3 \%$ \\
E7-2016 & 14 & 10.680 & $19 \%$ & 5.218 .273 & $15 \%$ & 2.591 .752 & $-3 \%$ \\
\hline E1-2017 & 15 & 8.870 & $7 \%$ & 40.419 & $4 \%$ & 3.246 .202 & $-2 \%$ \\
E2-2017 & 16 & 11.071 & $9 \%$ & 10.763 & $-53 \%$ & 6.242 .404 & $-2 \%$ \\
E3-2017 & 17 & 11.985 & $6 \%$ & 227.022 & $-20 \%$ & 3.196 .468 & $-4 \%$ \\
E4-2017 & 18 & 18.817 & $18 \%$ & 48.849 & $216 \%$ & 5.973 .483 & $-4 \%$ \\
E5-2017 & 19 & 35.575 & $-23 \%$ & 5.803 .061 & $-17 \%$ & 3.097 .234 & $-4 \%$ \\
E6-2017 & 20 & 23.723 & $43 \%$ & 759.900 & $44 \%$ & 7.061 .752 & $-6 \%$ \\
E7-2017 & 21 & 13.596 & $27 \%$ & 5.432 .087 & $4 \%$ & 2.495 .160 & $-4 \%$ \\
\hline & & & & & & & \\
\hline
\end{tabular}

Na Tabela 2, as DMUs encontram-se ordenadas de acordo com os respectivos valores de cada variável, iniciando pelos valores mais favoráveis em relação a cada variável. Para as variáveis OPEX e Emissões de GEE, que se deseja minimizá-los, iniciou-se da DMU com o menor valor para o maior dentre os registrados na Tabela 1 e para o Número de Consumidores, que se busca a maximização, do maior para o menor dos registrados na Tabela 1. O objetivo desta tabela será para auxiliar a explicar os resultados do índice de eficiência. Cada empresa encontra-se representada por uma cor diferente.

Tabela 2 - Ordenação das DMUS de acordo com as variáveis 


\begin{tabular}{|c|c|c|c|}
\hline Ordem & $\begin{array}{c}\text { OPEX } \\
\text { (MR\$) } \\
\text { menor --> maior }\end{array}$ & $\begin{array}{c}\text { EMISSOES GASES } \\
(\mathrm{tCO} 2 \mathrm{e}) \\
\text { menor }-->\text { maior }\end{array}$ & $\begin{array}{l}\text { CONSUMIDORES } \\
\text { (QUANT IDADE) } \\
\text { maior --> menor }\end{array}$ \\
\hline \multirow{10}{*}{ 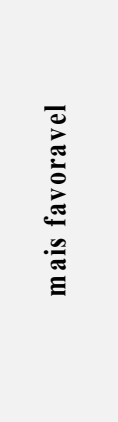 } & E1-2016 & E2-2015 & E6-2015 \\
\hline & E1-2017 & E2-2017 & E6-2016 \\
\hline & E7-2015 & E4-2016 & E6-2017 \\
\hline & E1-2015 & E2-2016 & E2-2015 \\
\hline & E2-2016 & E1-2015 & E2-2016 \\
\hline & E7-2016 & E1-2016 & E4-2015 \\
\hline & E2-2017 & E1-2017 & E2-2017 \\
\hline & E3-2016 & E4-2017 & E4-2016 \\
\hline & E3-2017 & E4-2015 & E4-2017 \\
\hline & E2-2015 & E3-2017 & E3-2015 \\
\hline \multirow[t]{3}{*}{ mediana } & E3-2015 & E3-2015 & E1-2015 \\
\hline & E7-2017 & E3-2016 & E5-2015 \\
\hline & E4-2016 & E6-2016 & E1-2016 \\
\hline \multirow{8}{*}{ 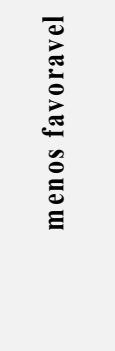 } & E6-2016 & E6-2017 & E3-2016 \\
\hline & E6-2015 & E6-2015 & E1-2017 \\
\hline & E4-2015 & E7-2015 & E5-2016 \\
\hline & E4-2017 & E7-2016 & E3-2017 \\
\hline & E6-2017 & E7-2017 & E5-2017 \\
\hline & E5-2015 & E5-2017 & E7-2015 \\
\hline & E5-2017 & E5-2016 & E7-2016 \\
\hline & E5-2016 & E5-2015 & E7-2017 \\
\hline
\end{tabular}

O modelo MORO-D será utilizado para avaliar as DMUs da Tabela 1, considerando três funções objetivo: (a) OPEX que é um input e será minimizado; (b) Emissões de gases, que também será minimizada, por se tratar de output indesejável, esse tratamento como insumo já foi aplicado em condições semelhantes no modelo MORO-D em [26]; e (c) Número de consumidores que se trata de output e será maximizado. A aplicação do modelo MOROD nesse caso segue a sua versão original, pois todas as variáveis avaliadas serão otimizadas. Será assumido retornos variáveis de escala (VRS), recomendado quando não se há certeza da existência de proporcionalidade entre as entradas e as saídas, e também por gerar uma fronteira de eficiência mais flexível.

\section{RESULTADOS E DISCUSSÃO}

Os resultados com a utilização do método MORO-D nos modelos desse estudo, foram obtidos com auxílio do software TRIMAP [27] e [28]. Os cálculos resultaram em 6 (seis) DMUs eficientes, correspondentes as empresas e anos (E2-2015), (E6-2015), (E12016), (E2-2016), (E6-2016) e (E2 - 2017), ou seja, em torno de 30\% do conjunto em estudo. Nota-se, que a empresa E2 obteve eficiência em todos os anos do período sob análise, o que pode ser explicado, visualizando a Tabela 2, por se destacar com os menores níveis de emissão de gases, além de apresentar nesses anos, número de consumidores e OPEX, próximos as empresas que lideram essas variáveis. Uma delas é justamente a E6 nos anos de 2015 e 2016, que se destaca no número de consumidores, e fica pouco abaixo da média de emissões de gases e OPEX. Finalmente a (E1- 2016), ocupa a melhor posição relativo à OPEX e na média em relação às demais. Portanto, nota-se que cada empresa eficiente, aparentemente obteve essa condição por se destacar em uma das variáveis. 
A seguir na Tabela 3 encontram-se listadas as DMUs ineficientes, e foi gerada a partir dos resultados obtidos com a utilização do método MORO-D nos modelos desse estudo, calculados com auxílio do citado software TRIMAP. Nessa Tabela consta o índice de eficiência calculado com a Equação (6), os benchmarks correspondentes à solução de cada índice de eficiência, as metas obtidas pela aplicação das Equações (4) e (5) utilizando os alvos dos referidos benchmarks e nas últimas colunas são apresentadas as variações entre as variáveis originais das DMUs ineficientes, listadas na Tabela 1, e suas metas constantes dessa Tabela 3.

Na referida Tabela 3, verifica-se que os resultados obtidos pelo modelo MORO-D, chegaram a ter de 3 a 7 alvos diferentes para cada uma das DMUs ineficientes, que segundo descrito em [13], tais variações quantitativas de resultados podem ocorrer por várias razões, mas sempre haverá pelo menos dois resultados. 
Tabela 3 - Indices de eficiencias, benchmarks, metas e variação valores originais - metas DMUs ineficientes

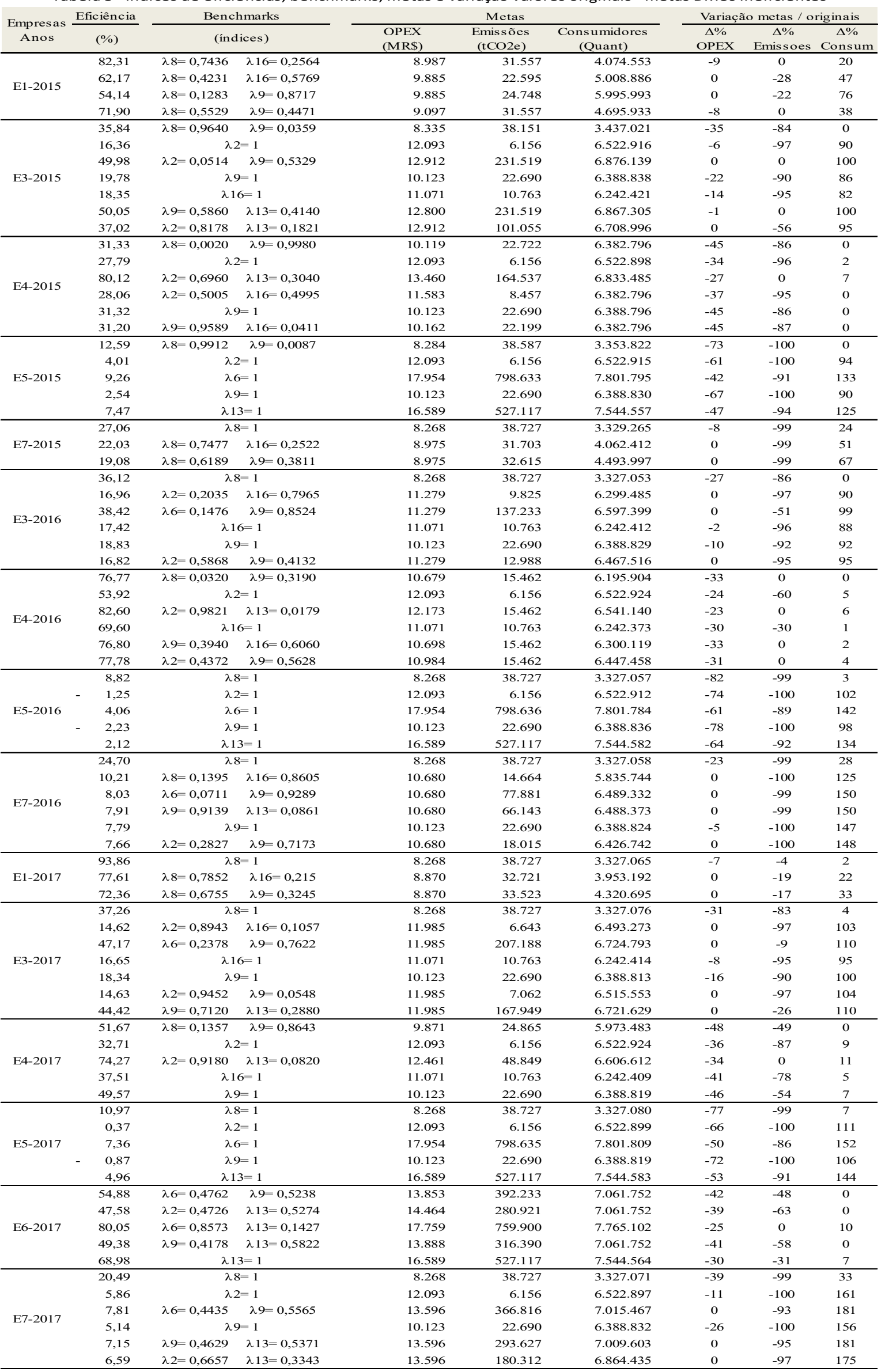


A escolha do alvo a ser seguido, para que as DMUs ineficientes se tornem eficientes, Angulo Meza et al. [12] sugerem quatro critérios que podem apoiar essa decisão. No primeiro critério a escolha da meta se faz pelo maior índice de eficiência, pois estes autores denotam que quanto maior o índice de eficiência da meta, mais próximo está de atingir a fronteira eficiente. E acrescentam, que quando não há limitações operacionais / gerenciais, a seleção do alvo com o maior índice de eficiência para a DMU é o recomendado. Esse critério é apresentado por eles como o critério do maior índice de eficiência. O $2^{\circ}$ critério tem como fundamento a possibilidade das empresas terem dificuldade de executarem um plano de melhoria baseado em múltiplos benchmarks. Assim, selecionando o alvo que tem o número mínimo de benchmarks, o plano de melhoria para a DMU seria mais fácil de determinar e executar. Esse critério é reportado no referido manuscrito como o número mínimo de critérios de referência. $\mathrm{O} 3^{\circ}$ critério consiste em selecionar um alvo que priorize uma das variáveis, de acordo com a preocupação da empresa, relacionada a uma das variáveis abordadas. Os referidos autores expõem que esse é o critério da priorização. Finalmente, eles apresentam o $4^{\circ}$ critério como trade-off, fundamentado na estimativa dos trade-offs entre as variáveis para apoiar a decisão. Os trade-offs são calculados dividindo as variações de uma variável em relação à variação de outra, apurando o impacto entre elas.

$\mathrm{Na}$ Tabela 4, encontram-se as DMUs ineficientes segundo o critério do maior índice de eficiência, com os respectivos benchmarks e as metas para tornar eficientes as DMUs ineficientes. Os dados foram estratificados da Tabela 3.

Tabela 4 - Maior índice de eficiência para as DMUs ineficientes

\begin{tabular}{|c|c|c|c|c|c|c|c|c|c|}
\hline \multirow[b]{2}{*}{$\begin{array}{c}\text { Empresas } \\
\text { Anos }\end{array}$} & \multirow{2}{*}{$\begin{array}{c}\text { Quant } \\
\text { Soluções }\end{array}$} & \multicolumn{2}{|c|}{ Maiores indices de eficiência } & \multicolumn{3}{|c|}{ Metas } & \multicolumn{3}{|c|}{ Variação (metas / originais) } \\
\hline & & $(\%)$ & benchmarks & $\begin{array}{l}\text { OPEX } \\
\text { (MR\$) }\end{array}$ & $\begin{array}{r}\text { Emissões } \\
(\mathrm{tCO} 2 \mathrm{e})\end{array}$ & $\begin{array}{c}\text { Consumidores } \\
\text { (Quant) }\end{array}$ & $\begin{array}{l}\% \% \\
\text { OPEX }\end{array}$ & $\begin{array}{c}\% \\
\text { Emissoes }\end{array}$ & $\begin{array}{c}\Delta \% \\
\text { Consum }\end{array}$ \\
\hline E1-2015 & 4 & 82,31 & 8 e 16 & 8.987 & 31.557 & 4.074 .553 & $-9 \%$ & $0 \%$ & $20 \%$ \\
\hline E3-2015 & 7 & 50,05 & 9 e 13 & 12.800 & 231.519 & 6.867 .305 & $-1 \%$ & $0 \%$ & $100 \%$ \\
\hline E4-2015 & 6 & 80,12 & 2 e 13 & 13.460 & 164.537 & 6.833 .485 & $-27 \%$ & $0 \%$ & $7 \%$ \\
\hline E5-2015 & 5 & 12,59 & 8 e 9 & 8.284 & 38.587 & 3.353 .822 & $-73 \%$ & $-100 \%$ & $0 \%$ \\
\hline E7-2015 & 3 & 27,06 & 8 & 8.268 & 38.727 & 3.329 .065 & $-8 \%$ & $-99 \%$ & $24 \%$ \\
\hline E3-2016 & 6 & 38,42 & 6 e 9 & 11.279 & 137.233 & 6.597 .399 & $0 \%$ & $-51 \%$ & $99 \%$ \\
\hline E4-2016 & 6 & 82,60 & 2 e 13 & 12.173 & 15.462 & 6.541 .140 & $-23 \%$ & $0 \%$ & $6 \%$ \\
\hline E5-2016 & 5 & 8,82 & 8 & 8.268 & 38.727 & 3.327 .057 & $-82 \%$ & $-99 \%$ & $3 \%$ \\
\hline E7-2016 & 6 & 24,70 & 8 & 8.268 & 38.727 & 3.327 .058 & $-23 \%$ & $-99 \%$ & $28 \%$ \\
\hline E1-2017 & 3 & 93,86 & 8 & 8.268 & 38.727 & 3.327 .065 & $-7 \%$ & $-4 \%$ & $2 \%$ \\
\hline E3-2017 & 7 & 47,17 & 6 e 9 & 11.985 & 207.188 & 6.724 .793 & $0 \%$ & $-9 \%$ & $110 \%$ \\
\hline E4-2017 & 5 & 74,27 & 2 e 13 & 12.461 & 48.849 & 6.606 .612 & $-34 \%$ & $0 \%$ & $11 \%$ \\
\hline E5-2017 & 5 & 10,97 & 8 & 8.268 & 38.727 & 3.327 .080 & $-77 \%$ & $-99 \%$ & $7 \%$ \\
\hline E6-2017 & 5 & 80,05 & 6 e 13 & 17.759 & 759.900 & 7.765 .102 & $-25 \%$ & $0 \%$ & $10 \%$ \\
\hline E7-2017 & 6 & 20,49 & 8 & 8.268 & 38.727 & 3.327 .071 & $-39 \%$ & $-99 \%$ & $33 \%$ \\
\hline
\end{tabular}

Constata-se pela Tabela 4, que a empresa E1 em 2017, apresenta as menores variações para se alcançar a eficiência, que pode ser explicado ao se verificar na Tabela 2, que ela possui o $2^{\circ}$ melhor valor de OPEX, ocupa posição favorável em emissões de gases, e pouco abaixo da média no número de consumidores. Por outro lado, conforme Tabela 3 , a empresa E5 em todos os anos, apresenta variações consideráveis em quase todas as variáveis para alcançar a meta e no conjunto possui as maiores variações, e tal fato pode ser justificado ao se evidenciar na Tabela 2 que esta empresa ocupa as últimas posições em valor de OPEX e emissões, ficando abaixo da média no número de consumidores, portanto explicando o baixo índice de performance. 
É importante ressaltar que algumas variações para alcançar a meta são de grande relevância, chegando à ordem de $100 \%$, o que a princípio não seria obtido em curto prazo, ou até mesmo seria viabilizado, portanto os níveis de variação devem ser analisados, se são exequíveis nas variáveis adotadas.

Esse fundamento fica evidente no caso em tela, ao se verificar na Tabela 1, as variações de um ano para outro no número de consumidores, que possui um patamar baixo, inclusive negativo a cada ano em todas as empresas. Pela característica deste indicador é improvável que ocorra grandes variações, pelo menos em curto prazo, pois o aumento ou queda dos consumidores neste segmento ocorre de forma paulatina. Da mesma forma para o OPEX, se verifica que a variação máxima foi de $48 \%$, que já é uma variação considerável, e está acima da média das demais variações, conforme Tabela 1. Quanto às emissões, elas estão sujeitas a variações maiores, como nos casos de escassez de recursos hídricos em determinadas regiões, quando há necessidade de aumentar a produção das termoelétricas locais, gerando maiores emissões de gases. Portanto pela Tabela 3, as DMUs que estariam dentro de um nível de exequibilidade com variação do número de consumidores e OPEX dentro da media de variação apresentada na Tabela 1, seriam as empresas E4 em todos os anos, E1 e E6 em 2017, encontram-se assinaladas em azul. Quanto à empresa E5 apesar de apresentar variação dentro do patamar do número de consumidores, no entanto possui variações muito grandes nas outras duas variáveis, o que também é aparentemente duvidoso que possa alcançar tais metas. Essas análises das soluções avaliando tais condições, proporcionam resultados realísticos de ocorrerem, inclusive poderiam ser restrições na modelagem.

A seguir a tabela 5, elencado as DMUs, de acordo com o segundo critério.

Tabela 5 - Número mínimo de referências para DMUs ineficientes

\begin{tabular}{|c|c|c|c|c|c|c|c|c|c|}
\hline \multirow{2}{*}{$\begin{array}{c}\text { Empresas } \\
\text { Anos }\end{array}$} & \multirow{2}{*}{$\begin{array}{c}\text { Quant } \\
\text { Soluções }\end{array}$} & \multicolumn{2}{|c|}{ Ìndices de eficiência } & \multicolumn{3}{|c|}{ Metas } & \multicolumn{3}{|c|}{ Variação (metas / originais) } \\
\hline & & $(\%)$ & benchmarks & $\begin{array}{l}\text { OPEX } \\
\text { (MR\$) } \\
\end{array}$ & $\begin{array}{r}\text { Emissões } \\
(\mathrm{tCO} 2 \mathrm{e}) \\
\end{array}$ & $\begin{array}{c}\text { Consumidores } \\
\text { (Quant) }\end{array}$ & $\begin{array}{l}\Delta \% \\
\text { OPEX }\end{array}$ & $\begin{array}{c}\Delta \% \\
\text { Emissoes }\end{array}$ & $\begin{array}{c}\Delta \% \\
\text { Consum. }\end{array}$ \\
\hline E1-2015 & 4 & 82,31 & 8 e 16 & 8.987 & 31.557 & 4.074 .553 & $-9 \%$ & $0 \%$ & $20 \%$ \\
\hline E3-2015 & 7 & 18,35 & 16 & 11.071 & 10.763 & 6.242 .421 & $-14 \%$ & $-95 \%$ & $82 \%$ \\
\hline E4-2015 & 6 & 31,32 & 9 & 10.123 & 22.690 & 6.388 .796 & $-45 \%$ & $-86 \%$ & $0 \%$ \\
\hline E5-2015 & 5 & 2,54 & 9 & 10.123 & 22.690 & 6.388 .830 & $-67 \%$ & $-100 \%$ & $90 \%$ \\
\hline E7-2015 & 3 & 27,06 & 8 & 8.268 & 38.727 & 3.329 .265 & $-8 \%$ & $-99 \%$ & $24 \%$ \\
\hline E3-2016 & 6 & 36,12 & 8 & 8.268 & 38.727 & 3.327 .053 & $-27 \%$ & $-86 \%$ & $0 \%$ \\
\hline E4-2016 & 6 & 69,60 & 16 & 11.071 & 10.763 & 6.242 .373 & $-30 \%$ & $-30 \%$ & $1 \%$ \\
\hline E5-2016 & 5 & 8,82 & 8 & 8.268 & 38.727 & 3.327 .057 & $-82 \%$ & $-99 \%$ & $3 \%$ \\
\hline E7-2016 & 6 & 24,70 & 8 & 8.268 & 38.727 & 3.327 .058 & $-23 \%$ & $-99 \%$ & $28 \%$ \\
\hline E1-2017 & 3 & 93,86 & 8 & 8.268 & 38.727 & 3.327 .065 & $-7 \%$ & $-4 \%$ & $2 \%$ \\
\hline E3-2017 & 7 & 16,65 & 16 & 11.071 & 10.763 & 6.242 .414 & $-8 \%$ & $-95 \%$ & $95 \%$ \\
\hline E4-2017 & 5 & 37,51 & 16 & 11.071 & 10.763 & 6.242 .409 & $-41 \%$ & $-78 \%$ & $5 \%$ \\
\hline E5-2017 & 5 & 10,97 & 8 & 8.268 & 38.727 & 3.327 .080 & $-77 \%$ & $-99 \%$ & $7 \%$ \\
\hline E6-2017 & 5 & 68,98 & 13 & 16.589 & 527.117 & 7.544 .564 & $-30 \%$ & $-31 \%$ & $7 \%$ \\
\hline E7-2017 & 6 & 20,49 & 8 & 8.268 & 38.727 & 3.327 .071 & $-39 \%$ & $-99 \%$ & $33 \%$ \\
\hline
\end{tabular}

$\mathrm{Na}$ Tabela 5, o critério de seleção dos alvos, corresponde ao número mínimo de critérios de referência. As DMUs que apresentaram mais de uma solução com única referência foi adotada a de maior índice de eficiência. A DMU 1 apresenta em todas as soluções duas referências, na tabela encontram-se as referências vinculadas ao maior índice de eficiência [12]. Nota-se também a ocorrência de grandes variações de algumas DMUs para atingir as respectivas metas, conforme assinalado em rosa na referida Tabela, que deve ser avaliada quanto a sua exequibilidade, segundo discutido anteriormente. 
$\mathrm{Na}$ Tabela 6 estão elencadas as soluções das DMUs ineficientes consoante à priorização do OPEX, ou seja, dentre os resultados de cada uma, selecionou-se o de menor valor para OPEX, corresponde ao terceiro critério descrito anteriormente. Nota-se uma predominância do benchmark da DMU 8 (eficiente), tal fato ocorre porque é a DMU que apresenta o menor valor de OPEX, portanto sendo a melhor referência. Da mesma forma assinalado em rosa as DMUs que possuem grandes variações para atingir as respectivas metas, que conforme já explanado deve ser avaliada a sua exequibilidade.

Tabela 6 - Priorizando redução do OPEX para DMUs ineficientes

\begin{tabular}{|c|c|c|c|c|c|c|c|c|c|}
\hline \multirow{2}{*}{$\begin{array}{c}\text { Empresas } \\
\text { Anos }\end{array}$} & \multirow{2}{*}{$\begin{array}{c}\text { Quant } \\
\text { Soluções }\end{array}$} & \multicolumn{2}{|c|}{ Ìndices de eficiência } & \multicolumn{3}{|c|}{ Metas } & \multicolumn{3}{|c|}{ Variação (metas / originais) } \\
\hline & & $(\%)$ & benchmarks & $\begin{array}{l}\text { OPEX } \\
\text { (MR\$) }\end{array}$ & $\begin{array}{r}\text { Emiss ões } \\
(\text { tCO2e })\end{array}$ & $\begin{array}{c}\text { Consumidores } \\
\text { (Quant) }\end{array}$ & $\begin{array}{l}\Delta \% \\
\text { OPEX }\end{array}$ & $\begin{array}{c}\Delta \% \\
\text { Emis soes }\end{array}$ & $\begin{array}{c}\Delta \% \\
\text { Consum }\end{array}$ \\
\hline E1-2015 & 4 & 82,31 & 8 e 16 & 8.987 & 31.557 & 4.074 .553 & $-9 \%$ & $0 \%$ & $20 \%$ \\
\hline E3-2015 & 7 & 35,84 & 8 e 9 & 8.335 & 38.151 & 3.437 .021 & $-35 \%$ & $-84 \%$ & $0 \%$ \\
\hline E4-2015 & 6 & 31,33 & 8 e 9 & 10.119 & 22.722 & 6.382 .796 & $-45 \%$ & $-86 \%$ & $0 \%$ \\
\hline E5-2015 & 5 & 12,59 & 8 e 9 & 8.284 & 38.587 & 3.353 .822 & $-73 \%$ & $-100 \%$ & $0 \%$ \\
\hline E7-2015 & 3 & 27,06 & 8 & 8.268 & 38.727 & 3.329 .265 & $-8 \%$ & $-99 \%$ & $24 \%$ \\
\hline E3-2016 & 6 & 36,12 & 8 & 8.268 & 38.727 & 3.327 .053 & $-27 \%$ & $-86 \%$ & $0 \%$ \\
\hline E4-2016 & 6 & 76,77 & 8 e 9 & 10.679 & 15.462 & 6.195 .904 & $-33 \%$ & $0 \%$ & $0 \%$ \\
\hline E5-2016 & 5 & 8,82 & 8 & 8.268 & 38.727 & 3.327 .057 & $-82 \%$ & $-99 \%$ & $3 \%$ \\
\hline E7-2016 & 6 & 24,70 & 8 & 8.268 & 38.727 & 3.327 .058 & $-23 \%$ & $-99 \%$ & $28 \%$ \\
\hline E1-2017 & 3 & 93,86 & 8 & 8.268 & 38.727 & 3.327 .065 & $-7 \%$ & $-4 \%$ & $2 \%$ \\
\hline E3-2017 & 7 & 37,26 & 8 & 8.268 & 38.727 & 3.327 .076 & $-31 \%$ & $-83 \%$ & $4 \%$ \\
\hline E4-2017 & 5 & 51,67 & 8 e 9 & 9.871 & 24.865 & 5.973 .483 & $-48 \%$ & $-49 \%$ & $0 \%$ \\
\hline E5-2017 & 5 & 10,97 & 8 & 8.268 & 38.727 & 3.327 .080 & $-77 \%$ & $-99 \%$ & $7 \%$ \\
\hline E6-2017 & 5 & 54,88 & 6 e 9 & 13.853 & 392.233 & 7.061 .752 & $-42 \%$ & $-48 \%$ & $0 \%$ \\
\hline E7-2017 & 6 & 20,49 & 8 & 8.268 & 38.727 & 3.327 .071 & $-39 \%$ & $-99 \%$ & $33 \%$ \\
\hline
\end{tabular}

Quanto ao quarto critério, para o caso em pauta, pode ser útil quantificar qual o impacto com o aumento do número de consumidores sobre os níveis de emissões ou do valor da OPEX, objetivando o gestor identificar seu alvo mais adequado. Para a apuração do tradeoff, foram selecionadas as DMUs ineficientes que tenham apresentado as menores variações para alcançar a meta nos três critérios anteriores, no caso DMUs (E4-2015), (E4-2016), (E1-2017), (E4-2017) e (E6-2017), sendo feita a apuração do trade-off entre o número de consumidores e o OPEX, obteve-se como resultado de menor impacto entre as variáveis da DMU (E4-2017), por apresentar mais homogeneidade, conforme se vislumbra no gráfico 1.

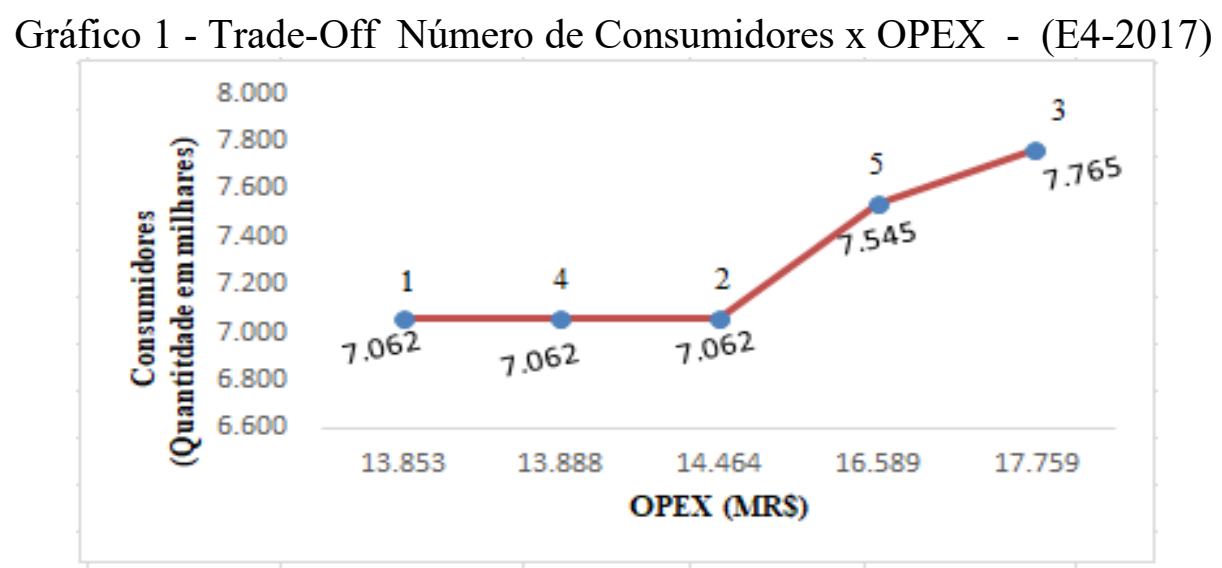

Conforme descrito em [12], o critério mais simples é usar o mais alto índice de eficiência, porque conforme visto anteriormente, é mais fácil de obtê-lo. Por outro lado, na prática, ao implementar um plano de melhorias, o número mínimo de critérios de referência é mais adequado. Isso acontece porque seguir um benchmark ou um mínimo número de 
benchmarks exige menos tempo e esforço do que o outro critério. Além disso, um desses critérios pode ser usado para todas as DMUs ineficientes, ou cada DMU poderia usar critérios diferentes, de acordo como entendimento do decisor. Pode ser possível usar um método de tomada de decisão multicritério quando a estrutura de preferência de uma empresa ou setor é conhecida. Isso exigiria uma seleção de metas viáveis para implementar e, em seguida, iniciar o processo com o método multicritério. Neste ponto, o conjunto de alvos poderia ser reduzido a apenas um ou dois alvos, então o esforço de usar um método multicritério seria reduzido. De qualquer forma, o uso de um método de tomada de decisão multicritério é mais adequado quando há um grande número de alvos em um conjunto para uma dada unidade produtora e sua estrutura de preferência é conhecida. Entretanto, conforme retratado por [12], as informações fornecidas pelos benchmarks não são usadas apenas para determinar os níveis de recursos, mas também para fornecer diretrizes para os seus procedimentos operacionais e gerenciais, servindo como um guia para ajustar os seus procedimentos e perseguir as melhores práticas.

\section{CONCLUSÕES}

O modelo utilizado permitiu fazer uma análise voltada para o campo da sustentabilidade das principais empresas do setor elétrico brasileiro, no ciclo de 3 anos, 2015 a 2017, com a aplicação da metodologia DEA multiobjetivo (MORO-D), a partir da otimização das variáveis econômica, social e ambiental, que correspondem a indicadores do GRI.

É importante ressaltar, que a aplicação de DEA na apuração de alternativas corporativas mais sustentáveis aliando os conceitos do TBL, econômico, social e ambiental, objetivando medir as respectivas eficiências de forma comparativa, poderá servir como referência, para as que não alcançaram o nível de excelência, revejam a sua gestão, empregando as melhores práticas, de tal forma que com os resultados obtidos possam promover melhorias futuras.

Essa busca por alternativas mais sustentáveis considerando diversos critérios quantitativos, no presente trabalho sob a perspectiva da aplicação multiobjetivo, privilegiouse o aumento do número de consumidores, a redução da emissão de gases do efeito estufa e da variável econômica OPEX, de cada DMU, que em resumo resultou como eficientes, cerca de $30 \%$ das DMUs analisadas. O método propiciou uma análise mais apurada sobre as melhores práticas a serem implementadas nas DMUs ineficientes, pois possibilitou selecionar resultados sob critérios distintos, flexibilizando a busca das empresas pelas melhores práticas, ao aplicar as alternativas de solução dos índices de benchmarks, inclusive quanto à realidade relativa ao que foi discutido sobre a exequibilidade de aplicação dos alvos.

Para execução dos cálculos dos modelos contemplando as 21 DMUs foi utilizado o software TRIMAP, que encontra soluções ótimas de Pareto para modelos multiobjetivo, com três funções objetivo, que corresponde à proposta deste trabalho. Com a aplicação do referido software sobre o modelo, foi obtido para cada DMU, as soluções das Funções Objetivo, os alvos para que as DMUs ineficientes se tornem eficientes, e propiciou o cálculo dos índices de eficiência das DMUs.

Esses resultados permitem aos gestores das empresas diante do cenário estudado, pensarem o que tem que ser melhorado para o futuro e o que deve ser proposto relativo ao contexto regulatório, econômico e climático que são relevantes para o setor conforme denotado nos relatórios analisados, bem como para os stakeholders decidirem em quais organizações vão investir seus recursos, se comprometerem ou se engajarem, facilitando a transparência para a tomada de decisão. 
$\mathrm{Na}$ expectativa de continuar a pesquisa no âmbito de problemas de cunho sustentável, sugere-se como trabalhos futuros a possibilidade de abranger o estudo com mais indicadores de sustentabilidade, e ainda, a possibilidade do uso conjunto de modelos avançados em DEA, que avaliem a produtividade dos fatores ao longo do tempo, observando as mudanças na tecnologia, como o uso do índice Malmquist.

\section{REFERÊNCIAS BIBLIOGRÁFICAS}

[1] PAZ, T. S. R; MACHADO, M. T. M.; LIMA, G. B. A.; ZOTES, L. P. Análise de Sustentabilidade com base no Princípio do Triple Bottom Line (TBL) pela Técnica de Similaridade com Solução Ideal (TOPSIS). XXXVIII SBPO - Simpósio Brasileiro de Pesquisa Operacional, Maceió, AL. 2018.

[2] CAIADO, R. G. G.; LEAL FILHO, W.; QUELHAS, O.L.G.; NASCIMENTO, D.L.M.; ÁVILA, L.V. A literature-based review on potentials and constraints in the implementation of the sustainable development goals. Journal of Cleaner Production, 2018.

[3] BRACCINI, A. M; MARGHERITA, E. G. Exploring Organizational Sustainability of Industry 4.0 under the Triple Bottom Line: The Case of a Manufacturing Company. Sustainability, v. 11, n. 1, p. 1-17, 2018.

[4] ELKINGTON, J. Partnerships from cannibals with forks: The triple bottom line of 21stcentury business. Environmental Quality Management, v. 8, n. 1, p. 37-51, 1998.

[5] CAIAdO, R. G. G.; LIMA, G. B. A.; GAVIÃO, L. O.; QUElHAS, O. L. G.; PASCHOALINO, F. F.. Sustainability Analysis in Electrical Energy Companies by Similarity Technique to Ideal Solution. IEEE Latin America Transactions, v. 15, n. 4, p. 675-681, 2017.

[6] Williams, J.; COFIElD, A. M.; EllisON, H.; JANSEN, F. A Pathway toward Widespread Sustainability Measurement and Communication. Sustainability: The Journal of Record, v. 3, n. 4, p. 235-240, 2010.

[7] BROWN, H. S.; JONG, M. e LEVY, D. L. Building institutions based on information disclosure: lessons from GRI's sustainability reporting. Journal of Cleaner Production. 17, 571-580, 2009.

[8] ZHOU, H. YANG, Y.; CHEN, Y.; ZHU, J.. Data envelopment analysis application in sustainability: The origins, development and future directions. European Journal of Operational Research, v. 264, n. 1, p. 1-16, 2018.

[9] CHARNES, A.; COOPER, W. W.; RHODES, E. Measuring the efficiency of decision making units. European Journal of Operational Research, v. 2, n. 6, p. 429-444, 1978.

[10] GOLANY, B.; ROLL, Y. An application procedure for DEA. Omega, v. 17, n. 3, p. $237-$ $250,1989$.

[11] LI, X. B.; REEVES, G. R. A multiple criteria approach to data envelopment analysis. European Journal of Operational Research, v. 115, n. 3, p. 507-517, 1999.

[12] ANGUlO MEZA, L; ARAYA, M. G.; IRIARTE, A.; ROBOLlEDO-LEIVA, R. A multiobjective DEA model to assess the eco-efficiency of agricultural practices within the CF+ DEA method. Computers and Electronics in Agriculture, 2018.

[13] QUARIGUASI FROTA NETO, J.; ANGULO MEZA, L. Alternative targets for data envelopment analysis through multi-objective linear programming: Rio de Janeiro 
Odontological Public Health System Case Study. Journal of the Operational Research Society, v. 58, n. 7, p. 865-873, 2007.

[14] GOMES JUNIOR, S. F.; SOARES DE MELlO, J. C. C. B.; ÂNGULO MEZA, L. DEA nonradial efficiency based on vector properties. International Transactions in Operational Research, v. 20, n. 3, p. 341-364, 2013.

[15] FARRELL, M. J. The measurement of productive efficiency. Journal of the Royal Statistical Society: Series A (General), 120(3), 253-281, 1957.

[16] ZHOU, P.; BENG, W. A. Linear programming models for measuring economy-wide energy efficiency performance. Energy Policy 36.8: 2911-2916, 2008.

[17] BANKER, R. D.; CHARNES, A.; COOPER, W. W. Some models for estimating technical and scale inefficiencies in data envelopment analysis. Management Science, 30(9): 10781092, 1984.

[18] DJORDJEVIĆ, B.; KRMAC, E.; MLINARIĆ, T. J. Non-radial DEA model: A new approach to evaluation of safety at railway level crossings. Safety Science, 103: 234-246, 2018.

[19] OSMAN, I. H.; ANOUZE, A. L.; IRANI, Z.; AL-AYOUBI, B.; LEE, H.; BALCI, A.; MEDENI, T. D.; WEERAKKODY, V. C. Framework to evaluate e government services: A citizen-centric perspective. Government information quarterly, 31(2), 243-256, 2014.

[20] SCHEEL, H. Undesirable outputs in efficiency valuations. European Journal of Operational Research, vol. 132, pp. 400-410, 2009.

[21] TSCHAFFON, P. B.; ANGULO MEZA, L. Assessing the Efficiency of the Electric Energy Distribution using Data Envelopment Analysis with undesirable outputs. IEEELatin America Transactions, vol. 12, n. 6. pp. 1027-1035, 2014.

[22] LINS, M. P. E.; ANGULO MEZA, L.; SILVA, A. C. M. da. A multi-objective approach to determine alternative targets in data envelopment analysis. Journal of the Operational Research Society, 55 (10), 1090-1101, 2004.

[23] BMFBOVESPA. Índice de Sustentabilidade Empresarial (ISE). $<\underline{\text { http://www.bmfbovespa.com.br/pt br/produtos/indices/indices-de-sustentabilidade/ }}$ indice-de-sustentabilidade-empresarial-ise.htm $>$ (Acessado em 10/12/2018).

[24] DAPPER, S. N.; SPOHR, C.; ZANINI, R. R. Poluição do ar como fator de risco para a saúde: uma revisão sistemática no Estado de São Paulo. Estudos Avançados, 30 (86), 2014.

[25] ANEEL - Agência Nacional de Energia Elétrica. Indicadores. $<\underline{\text { http://www2.aneel.gov.br/ }}$ cedoc/_Proret_Submod_2_2_v3.pdf.> (Acessado em 25/11/2018).

[26] LOZANO, S.; IRIBARREN, D.; MOREIRA, M.T.; FEIJOO, G. The link between operational efficiency and environmental impacts: a joint application of life cycle assessment and data envelopment analysis. Science of the Total Environment, v. 407, n. 5, p. 1744-1754, 2009.

[27] CLÍMACO, J. C. N.; ANTUNES, C. H. TRIMAP - an interactive tricriteria linear programming package. Found. Control Eng., v. 12, n. 3, p. 101-119, 1987.

[28] CLÍMACO J. C. N.; ANTUNES, C. H. Implementation of a user-friendly software package - A guided tour of TRIMAP. In: Models and Methods in Multiple Criteria Decision Making. Pergamon, p. 1299-1309, 1989. 